\title{
Homogenization of variational inequalities for the $p$-Laplace operator in perforated media along manifolds
}

\author{
D. Gómez ${ }^{a}$, E. Pérez $^{b, \star}$, A.V. Podolskii ${ }^{c}$, T.A. Shaposhnikova ${ }^{c}$
}

(a) Departamento de Matemáticas, Estadística y Computación, Universidad de Cantabria, Avenida de los Castros s/n, 39005 Santander, Spain; gomezdel@unican.es

(b) Departamento de Matemática Aplicada y Ciencias de la Computación, Universidad de Cantabria, Avenida de los Castros s/n, 39005 Santander, Spain; meperez@unican.es

(c) Department of Differential Equations, Moscow State University, Leninskie Gory, 119991, GSP-2, Moscow, Russia; originalea@ya.ru, shaposh.tan@mail.ru

$(\star)$ Corresponding author

Abstract: We address homogenization problems of variational inequalities for the $p$-Laplace operator in a domain of $\mathbb{R}^{n}(n \geq 3, p \in[2, n))$ periodically perforated by balls of radius $O\left(\varepsilon^{\alpha}\right)$ where $\alpha>1$ and $\varepsilon$ is the size of the period. The perforations are distributed along a $(n-1)$ dimensional manifold $\gamma$, and we impose constraints for solutions and their fluxes (associated with the $p$-Laplacian) on the boundary of the perforations. These constraints imply that the solution is positive and that the flux is bounded from above by a negative, nonlinear monotonic function of the solution multiplied by a parameter $\varepsilon^{-\kappa}, \kappa \in \mathbb{R}$ and $\varepsilon$ is a small parameter that we shall make to go to zero. We analyze different relations between the parameters $p, n, \varepsilon, \alpha$ and $\kappa$, and obtain homogenized problems which are completely new in the literature even for the case $p=2$.

AMS Mathematics Subject Classification: 35B27, 35J60, 35J87, 35B25.

\section{Introduction}

In this paper, we study the asymptotic behavior of the solution $u_{\varepsilon}$ of a variational inequality for the $p$-Laplace operator posed in a domain $\Omega_{\varepsilon}$ which is obtained by removing small balls $G_{\varepsilon}$ of diameter $O\left(\varepsilon^{\alpha}\right)$ from a fixed domain $\Omega$ of $\mathbb{R}^{n}$; here $n \geq 3, p \in[2, n)$ and $\alpha>1$. These balls, the cavities, are periodically distributed along a $(n-1)$-dimensional manifold $\gamma$ at a distance $\mathrm{O}(\varepsilon)$ between them, $\varepsilon$ being a small parameter that we shall make to go to 0 (see Figure 1). On the boundary of the cavities $S_{\varepsilon}$, we consider the nonlinear 
restrictions $u_{\varepsilon} \geq 0, \partial_{\nu_{p}} u_{\varepsilon} \geq-\varepsilon^{-\kappa} \sigma\left(x, u_{\varepsilon}\right)$, and $u_{\varepsilon}\left(\partial_{\nu_{p}} u_{\varepsilon}+\varepsilon^{-\kappa} \sigma\left(x, u_{\varepsilon}\right)\right)=0$ which involve the so-called adsorption parameter $\varepsilon^{-\kappa}$ with $\kappa \in \mathbb{R}$ and the function $\sigma$. Here $\sigma=\sigma(x, u)$ is a continuously differentiable function defined in $\bar{\Omega} \times \mathbb{R}$, strictly monotone increasing with respect to $u$, and $\partial_{\nu_{p}} u$ denotes $|\nabla u|^{p-2}(\nabla u, \nu)$ where $\nu$ is the unit outward normal vector to the boundary of $\Omega_{\varepsilon}$. Also $u_{\varepsilon}$ satisfies a Dirichlet condition on $\partial \Omega$.

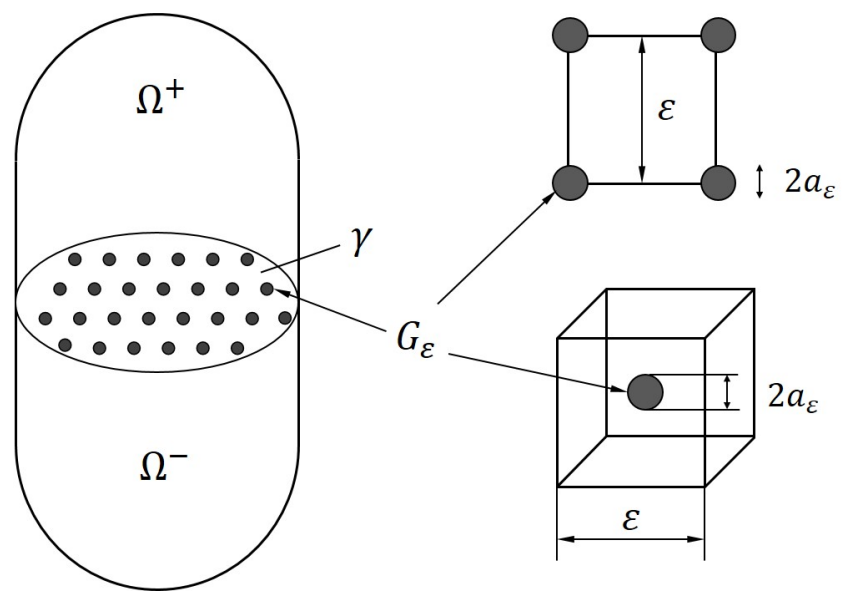

Figure 1: The geometrical configuration of $\Omega_{\varepsilon}$ and the periodicity cell.

The aim of this paper is to describe all the possible homogenized models depending on the parameters of the problem, $p, n, \varepsilon, \alpha$ and $\kappa$, and more precisely to characterize the possible relations between the parameters which give rise to a new term in the homogenized problem, the so-called strange term in the literature of homogenization problems (cf. $[2,14,15,8]$ for terminology, related problems and further references). In fact, when computing the homogenized problem, we obtain critical sizes of the perforations and critical relations for the adsorption in such a way that, depending on whether these relations are satisfied, the strange term can change its character or it may disappear. In any case, since we deal with a boundary homogenization problem, the strange term only appears in the conditions on the manifold $\gamma$.

The classical critical size of the perforations is given by $\alpha=(n-1) /(n-p)$ while $\kappa=(\alpha-1)(n-1)$ is referred to as critical relation for the adsorption parameter. It should be noted that this last critical relation implies that the total area of the perforations multiplied by the adsorption parameter is of order $O(1)$ (cf. Figure 2). In addition, depending on the value of the parameters $\alpha$ and $\kappa$, the homogenized problem can change strongly its character ranging from boundary value problems (cf. (11)-(12), (50) and (51)) to unilateral problems (cf. (47)-(48)). See Figure 2 for a sketch of all the homogenized problems. We emphasize that most of these homogenized problems appear for the first time in the literature. Even when $p$ is equal to 2, models (11) and (47) are not considered in the literature for the nonlinear functions $g$ and $h$ given by (12b), (12c) and (48). 


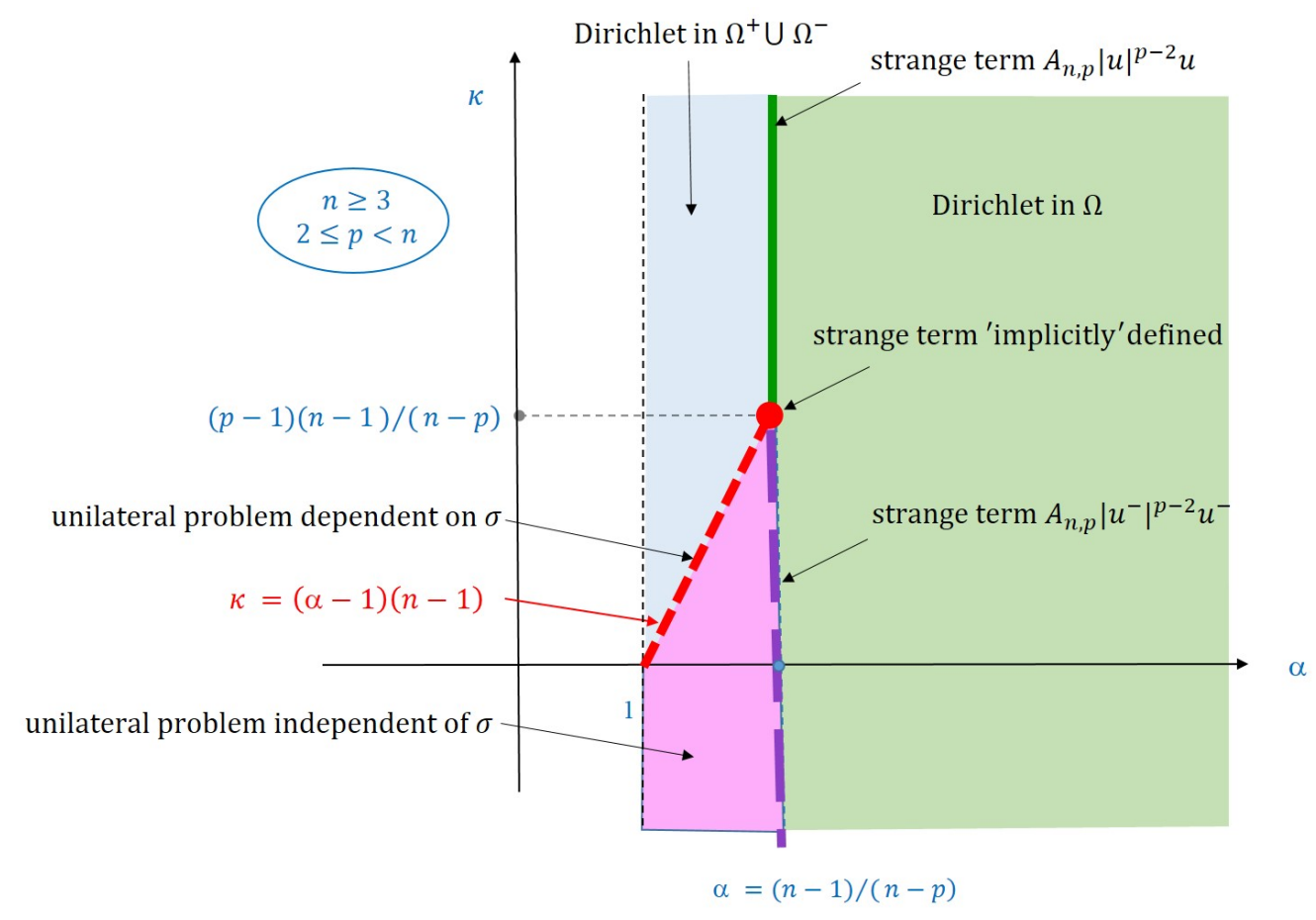

Figure 2: Sketch of homogenized problems depending on the relations between $\alpha$ and $\kappa$ $(n$ and $p$ fixed).

Taking into account the general idea of this paper for the $p$-Laplacian, the constraints and the technique, the closest paper in the literature is [5]. In spite of this, [5] deals with different geometrical configuration since the cavities are distributed over the whole domain: cf. also $[2,3,13,14,15,20,22,24]$ for perforated domains over the whole volume. However, we observe that most of the references are related with cavities over manifolds as we consider here. In fact, similar geometrical configurations for linear and nonlinear boundary value problems, as well as for variational inequalities, have been considered in many previous papers for operators different from the $p$-Laplacian (cf. $[1,6,7,8,9,16$, 17, 23] for further references). Comparing with the present paper, [23] studies variational inequalities for the biharmonic operator; $[1,8,9,16,17]$ consider the Laplace operator and linear problems; [1] contains an extra advection term related with the flow velocity; [6, 7] consider variational inequalities for the Laplace operator for certain relations between $\kappa$ and $\alpha$. [17] considers a boundary value problem for the $p$-Laplacian for the particular relation $\alpha=(n-1) /(n-p)$ and $\kappa=(p-1)(n-1) /(n-p)$. Here, we complete the results in $[6,7]$ when $p=2$ and extend them for the $p$-Laplacian and for all the possible relations between parameters. We refer to $[5,7]$ for an extensive bibliography on variational inequalities in homogenization problems and to $[4,5]$ for that on applications of the $p$-Laplacian to different models arising, e.g., in Newtonian fluids, glaciology and 
flows through porous media.

The problem under consideration (4) can arise in the framework of the modelling of the diffusion of substances in porous media, and more specifically, in nonlinear diffusion models in which an adsorption phenomenon can occur on the boundary of the perforations $S_{\varepsilon}$. Here we assume a strongly nonlinear adsorption law with a large parameter: see Remark 1 in this connection. The solution $u_{\varepsilon}$ represents, e.g., the concentration of polluting substance in a fluid, in a stationary regime, where also we have assumed that there is no influence of the fluid. See $[1,10,11,12,18,19,21]$ and references therein for more realistic models: time dependent models, models with an advection term linking the velocity of the fluid and the concentration, or models with additional unknowns or reactive terms arising in the partial differential equation and on the boundary conditions. However, we obtain strong convergence results (cf. Theorems 1-4) which will likely be weakened when dealing with more complex models. See $[1,8,9]$ for different models with the geometrical configuration here considered (cf. Figure 1) and $p=2$.

As regards the structure of the paper: Section 2 contains the setting of the $\varepsilon$-dependent problem. Section 3 provides the convergence results and their proofs for the critical size of the perforations (cf. the vertical line $\alpha=(n-1) /(n-p)$ in Figure 2). To avoid repetitions, we gather these results in the statement of Theorem 1. Section 4 addresses the rest of the cases: see Theorems 2, 3 and 4. For the sake of brevity, we sketch out their proofs here: we refer to $[5,7,8]$ for further details. Finally, for the sake of completeness, in the Appendix we introduce some auxiliary results useful for proofs.

\section{Statement of the problem}

Let $\Omega$ be a bounded domain in $\mathbb{R}^{n}, n \geq 3$, with a smooth boundary $\partial \Omega$. Assume that $\gamma=\Omega \cap\left\{x_{1}=0\right\} \neq \emptyset$ is a domain on the hyperplane $\left\{x_{1}=0\right\}$. Let $\varepsilon$ be a small positive parameter that we shall make converge towards zero. We set $\widetilde{\Omega}_{\varepsilon}=\{x \in \Omega: \rho(x, \partial \Omega)>$ $2 \varepsilon\}$ where $\rho$ denotes the distance.

We denote by $G_{0}$ the ball of radius 1 centered at the origin of coordinates. Let $\omega_{n}$ be the area of the unit sphere in $\mathbb{R}^{n}$, that is, $\omega_{n}=\left|\partial G_{0}\right|$. For a domain $B$ and for $\delta>0$, we define the sets $\delta B=\left\{x: \delta^{-1} x \in B\right\}$. We set

$$
G_{\varepsilon}=\bigcup_{j \in \Upsilon_{\varepsilon}}\left(a_{\varepsilon} G_{0}+\varepsilon j\right)=\bigcup_{j \in \Upsilon_{\varepsilon}} G_{\varepsilon}^{j}
$$

where $\Upsilon_{\varepsilon}=\left\{j \in \mathbb{Z}^{\prime}:\left(a_{\varepsilon} G_{0}+\varepsilon j\right) \cap \widetilde{\widetilde{\Omega}}_{\varepsilon} \neq \emptyset\right\}, \mathbb{Z}^{\prime}$ is the set of vectors of the form $z=\left(0, z_{2}, \ldots, z_{n}\right)$ with integer components $z_{j}, j=2, \ldots, n, a_{\varepsilon}=C_{0} \varepsilon^{\alpha}, C_{0}>0$ and $\alpha>1$ (see Figure 1). Obviously, we have $\left|\Upsilon_{\varepsilon}\right|=O\left(\varepsilon^{1-n}\right)$. Now, we can define

$$
\Omega_{\varepsilon}=\Omega \backslash \overline{G_{\varepsilon}}, S_{\varepsilon}=\partial G_{\varepsilon}, \partial \Omega_{\varepsilon}=\partial \Omega \cup S_{\varepsilon} .
$$


We consider the space $W^{1, p}\left(\Omega_{\varepsilon}, \partial \Omega\right)\left(W^{1, p}(\Omega, \partial \Omega)\right.$, respectively) to be the completion with respect to $W^{1, p}\left(\Omega_{\varepsilon}\right)$-norm $\left(W^{1, p}(\Omega)\right.$-norm, respectively) of the set of infinitely differentiable functions in $\bar{\Omega}_{\varepsilon}(\bar{\Omega}$, respectively), vanishing in a neighborhood of $\partial \Omega$.

Let us consider $\sigma(x, u)$ a continuously differentiable function of variables $(x, u) \in \bar{\Omega} \times \mathbb{R}$ satisfying:

$$
\begin{gathered}
\sigma(x, 0)=0 \\
(\sigma(x, u)-\sigma(x, v))(u-v) \geq k_{1}|u-v|^{p}
\end{gathered}
$$

and

$$
|\sigma(x, u)| \leq k_{2}|u|^{p-1}
$$

for all $x \in \bar{\Omega}, u, v \in \mathbb{R}$, and certain constants $k_{1}>0, k_{2}>0$.

For $f \in L^{q}(\Omega)$ with $q=p /(p-1)$, we consider the following problem

$$
\left\{\begin{array}{l}
-\Delta_{p} u_{\varepsilon}=f \quad \text { in } \Omega_{\varepsilon}, \quad u_{\varepsilon}=0 \quad \text { on } \partial \Omega \\
u_{\varepsilon} \geq 0, \quad \partial_{\nu_{p}} u_{\varepsilon} \geq-\varepsilon^{-\kappa} \sigma\left(x, u_{\varepsilon}\right), \quad u_{\varepsilon}\left(\partial_{\nu_{p}} u_{\varepsilon}+\varepsilon^{-\kappa} \sigma\left(x, u_{\varepsilon}\right)\right)=0 \quad \text { for } x \in S_{\varepsilon}
\end{array}\right.
$$

where $\Delta_{p} u \equiv \operatorname{div}\left(|\nabla u|^{p-2} \nabla u\right), \partial_{\nu_{p}} u \equiv|\nabla u|^{p-2}(\nabla u, \nu), \nu$ denotes the unit outward normal to $\Omega_{\varepsilon}$ on $S_{\varepsilon}$ and $\kappa \in \mathbb{R}$.

The variational formulation of problem (4) is: find $u_{\varepsilon} \in K_{\varepsilon}$ satisfying

$$
\int_{\Omega_{\varepsilon}}\left|\nabla u_{\varepsilon}\right|^{p-2} \nabla u_{\varepsilon} \nabla\left(\psi-u_{\varepsilon}\right) d x+\varepsilon^{-\kappa} \int_{S_{\varepsilon}} \sigma\left(x, u_{\varepsilon}\right)\left(\psi-u_{\varepsilon}\right) d s \geq \int_{\Omega_{\varepsilon}} f\left(\psi-u_{\varepsilon}\right) d x, \quad \forall \psi \in K_{\varepsilon}
$$

where the set $K_{\varepsilon}$ is defined by

$$
K_{\varepsilon}=\left\{g \in W^{1, p}\left(\Omega_{\varepsilon}, \partial \Omega\right): g \geq 0 \text { a.e. on } S_{\varepsilon}\right\} \text {. }
$$

The existence and uniqueness of the solution $u_{\varepsilon} \in K_{\varepsilon}$ of problem (5)-(6) follows from the monotonicity of the functions $|u|^{p-2} u$ and $\sigma(x, u)$ with respect to $u$ (cf. (2)). In addition, $u_{\varepsilon} \in K_{\varepsilon}$ also satisfies the inequality

$$
\int_{\Omega_{\varepsilon}}|\nabla \psi|^{p-2} \nabla \psi \nabla\left(\psi-u_{\varepsilon}\right) d x+\varepsilon^{-\kappa} \int_{S_{\varepsilon}} \sigma(x, \psi)\left(\psi-u_{\varepsilon}\right) d s \geq \int_{\Omega_{\varepsilon}} f\left(\psi-u_{\varepsilon}\right) d x, \quad \forall \psi \in K_{\varepsilon}:
$$

see, e.g., [4] or [5] for the technique.

Let us denote by $\mathcal{P}_{\varepsilon} u_{\varepsilon}$ an extension of $u_{\varepsilon}$ to $\Omega, \mathcal{P}_{\varepsilon} u_{\varepsilon} \in W^{1, p}(\Omega, \partial \Omega)$, satisfying:

$$
\left\|\mathcal{P}_{\varepsilon} u_{\varepsilon}\right\|_{W^{1, p}(\Omega)} \leq K\left\|u_{\varepsilon}\right\|_{W^{1, p}\left(\Omega_{\varepsilon}\right)}, \quad\left\|\nabla \mathcal{P}_{\varepsilon} u_{\varepsilon}\right\|_{L^{p}(\Omega)} \leq K\left\|\nabla u_{\varepsilon}\right\|_{L^{p}\left(\Omega_{\varepsilon}\right)} .
$$

The construction of such an extension can be done with the technique in Theorem 2 of [22]. Taking into account (8) and the properties of the function $\sigma(x, u)$, we set in (5) $\psi \equiv 0$ and we obtain the following estimate for the solution $u_{\varepsilon}$ :

$$
\left\|\mathcal{P}_{\varepsilon} u_{\varepsilon}\right\|_{W^{1, p}(\Omega)}^{p}+\varepsilon^{-\kappa}\left\|u_{\varepsilon}\right\|_{L^{p}\left(S_{\varepsilon}\right)}^{p} \leq K\|f\|_{L^{q}\left(\Omega_{\varepsilon}\right)}^{q} .
$$


Thus, for each sequence, there is a subsequence (still denoted by $\varepsilon$ ) such that, as $\varepsilon \rightarrow 0$,

$$
\mathcal{P}_{\varepsilon} u_{\varepsilon} \rightarrow u \text { in } W^{1, p}(\Omega, \partial \Omega) \text { - weak and } \mathcal{P}_{\varepsilon} u_{\varepsilon} \rightarrow u \text { in } L^{p}(\Omega),
$$

for a certain function $u$ which, once identified, provides the convergences (10) for the whole sequence of $\varepsilon$.

Throughout the rest of the paper, we show that this limit function $u$ is the unique solution of a homogenized problem which depends on the relation between the parameters $n, p, \alpha$ and $\kappa$. That is, depending on the dimension of the space, the value of $p$, and the different relations between the $\varepsilon$-dependent parameters (the radius of the cavities $O\left(\varepsilon^{\alpha}\right)$ and the adsorption parameter $O\left(\varepsilon^{-\kappa}\right)$ ), we have very different limit behaviors for the solution of problem (4). As a matter of fact, among all the relations, we highlight two critical relations: the critical size of the perforations given by $\alpha=(n-1) /(n-p)$ and the critical relation for the adsorption parameter given by $\kappa=(\alpha-1)(n-1)$. The first relation leads us to three different strange terms in the transmission condition on $\gamma$ for the homogenized problem: see Section 3. The second relation, namely the case where $\left|S_{\varepsilon}\right| \varepsilon^{-\kappa}=O(1)$, can also provide a strange term: see Section 4 . The relation $\alpha=(n-1) /(n-p)$ and $\kappa=(\alpha-1)(n-1)=(n-1)(p-1) /(n-p)$ is referred to as the most critical case (cf. problem (11),(12a)). We consider the nine possibilities for the couple of parameters $\alpha$ and $\kappa$ which can be larger than, equal to or smaller than the critical sizes, and we obtain seven possible different limit behaviors of $\mathcal{P}_{\varepsilon} u_{\varepsilon}$ (see Figure 2). The results obtained are in good agreement with those found in the literature for the Laplace operator (see [6] and [7]).

In what follows, we denote by $\Omega^{-}$and $\Omega^{+}$the domains $\Omega^{-}=\Omega \cap\left\{x_{1}<0\right\}$ and $\Omega^{+}=\Omega \cap\left\{x_{1}>0\right\}$ respectively. Also, the brackets mean $\left.[g]\right|_{P \in \gamma}=\lim _{p \rightarrow P, p \in \Omega^{+}} g(p)-$ $\lim _{p \rightarrow P, p \in \Omega^{-}} g(p)$ for any point $P \in \gamma$. Moreover, for a function $u$ in $W^{1, p}(\Omega), u^{+}$and $u^{-}$ denote $u^{+}=\sup (u(x), 0)$ and $u^{-}=u-u^{+}$respectively.

\section{Critical size for perforations}

In this section, we consider the critical size for perforations, that is, $\alpha=(n-1) /(n-p)$. The homogenized problem is the boundary value problem (11) where $g$ is the function defined by (12), which depends on the value of the parameter $\kappa$. In the most critical case, $\kappa=(n-1)(p-1) /(n-p)$, the strange term is the sum of two terms related to the contribution of both restrictions $u_{\varepsilon} \geq 0$ and $\partial_{\nu_{p}} u_{\varepsilon} \geq-\varepsilon^{-\kappa} \sigma\left(x, u_{\varepsilon}\right)$ on the boundary of the perforations; one of the terms includes a function defined implicitly from the functional equation (13) and the other one is of the type $\left|u^{-}\right|^{p-2} u^{-}$(cf. (12a)). Also, an averaged constant appears multiplying these terms. When $\alpha=(n-1) /(n-p)$ and $\kappa \neq(n-$ 
1) $(p-1) /(n-p)$, the homogenized problem does not depend on the function $\sigma$ and the reaction term is of the type $\left|u^{-}\right|^{p-2} u^{-}$or $|u|^{p-2} u$, multiplied by an averaged constant, depending on whether the adsorption is small or large, respectively (conform to (12b) or (12c), respectively).

Theorem 1. Let $\alpha=(n-1) /(n-p)$ with $n \geq 3, p \in[2, n)$, and let $u_{\varepsilon}$ be the weak solution of (4). Then, the limit of $\mathcal{P}_{\varepsilon} u_{\varepsilon}$ in (10), u, is the weak solution of problem

$$
\left\{\begin{array}{l}
-\Delta_{p} u=f \quad \text { in } \Omega^{+} \cup \Omega^{-}, \quad u=0 \quad \text { on } \partial \Omega, \\
{[u]=0, \quad\left[|\nabla u|^{p-2} \partial_{x_{1}} u\right]=g \quad \text { on } \gamma,}
\end{array}\right.
$$

where the function $g$ is given by

$$
\begin{array}{ll}
g(x, u)=\mathcal{A}_{n, p}\left(\left|H\left(x, u^{+}\right)\right|^{p-2} H\left(x, u^{+}\right)+\left|u^{-}\right|^{p-2} u^{-}\right) & \text {if } \kappa=\frac{(n-1)(p-1)}{n-p}, \\
g(x, u)=\mathcal{A}_{n, p}\left|u^{-}\right|^{p-2} u^{-} & \text {if } \kappa<\frac{(n-1)(p-1)}{n-p}, \\
g(x, u)=\mathcal{A}_{n, p}|u|^{p-2} u & \text { if } \kappa>\frac{(n-1)(p-1)}{n-p},
\end{array}
$$

$\mathcal{A}_{n, p}=\left(\frac{n-p}{p-1}\right)^{p-1} C_{0}^{n-p} \omega_{n}$ and, for every $(x, \tau) \in \Omega \times \mathbb{R}, H(x, \tau)$ is the solution of the functional equation

$$
\mathcal{D}_{n, p}|H|^{p-2} H=\sigma(x, \tau-H)
$$

with $\mathcal{D}_{n, p}=\left(\frac{n-p}{p-1}\right)^{p-1} C_{0}^{1-p}$.

Proof. We divide the proof in three parts. In the first one, we show the result for $\kappa=$ $(n-1)(p-1) /(n-p)$. In the second one and the third one, we do it for $\kappa$ less or greater than $(n-1)(p-1) /(n-p)$, respectively.

First step: $\kappa=(n-1)(p-1) /(n-p)$

The variational formulation of (11) reads: find $u \in W^{1, p}(\Omega, \partial \Omega)$ such that

$$
\int_{\Omega}|\nabla u|^{p-2} \nabla u \nabla \phi d x+\int_{\gamma} g(x, u) \phi d \hat{x}=\int_{\Omega} f \phi d x, \quad \forall \phi \in W^{1, p}(\Omega, \partial \Omega),
$$

where $\hat{x}=\left(x_{2}, \ldots, x_{n}\right)$. Let us note that on account of Lemma 1 , the function $H(x, u)$ arising in (12a) is a well defined function satisfying $H(x, 0)=0,(52)$ and (53). From the monotonicity of the functions $|z|^{p-2} z$ and $|H(x, z)|^{p-2} H(x, z)$ with respect to $z$ (see (52)), $p \geq 2$, the existence and uniqueness of solution of (14) holds for the function $g$ given by (12a): see, e.g., [7] and [8] for related problems.

Below, we construct the test functions (19) which allow us to pass to the limit in (7), as $\varepsilon \rightarrow 0$. Let us denote by $P_{\varepsilon}^{j}$ the center of the ball $G_{\varepsilon}^{j}, j \in \Upsilon_{\varepsilon}$. We denote by $T_{\varepsilon / 4}^{j}$ the ball of radius $\varepsilon / 4$ with center $P_{\varepsilon}^{j}$. Let $w_{\varepsilon}^{j}$ be the solution of the following problem

$$
\Delta_{p} w_{\varepsilon}^{j}=0 \text { in } T_{\varepsilon / 4}^{j} \backslash \overline{G_{\varepsilon}^{j}}, \quad w_{\varepsilon}^{j}=1 \text { on } \partial G_{\varepsilon}^{j}, \quad w_{\varepsilon}^{j}=0 \text { on } \partial T_{\varepsilon / 4}^{j} .
$$


It can easily be verified that for $p \in[2, n)$ we have

$$
w_{\varepsilon}^{j}(x)=\frac{\left|x-P_{\varepsilon}^{j}\right|^{(p-n) /(p-1)}-\left(\frac{\varepsilon}{4}\right)^{(p-n) /(p-1)}}{a_{\varepsilon}^{(p-n) /(p-1)}-\left(\frac{\varepsilon}{4}\right)^{(p-n) /(p-1)}} .
$$

We define the function $W_{\varepsilon} \in W^{1, p}(\Omega, \partial \Omega)$ by setting

$$
W_{\varepsilon}(x)=w_{\varepsilon}^{j}(x), \quad x \in T_{\varepsilon / 4}^{j} \backslash \overline{G_{\varepsilon}^{j}}, j \in \Upsilon_{\varepsilon},
$$

extended by 1 inside $G_{\varepsilon}^{j}, j \in \Upsilon_{\varepsilon}$, and by 0 in $\mathbb{R}^{n} \backslash \bigcup_{j \in \Upsilon_{\varepsilon}} \overline{T_{\varepsilon / 4}^{j}}$. Thus, we compute

$$
\begin{gathered}
\left\|W_{\varepsilon}\right\|_{L^{m}(\Omega)}^{m} \leq K\left(\varepsilon^{1+(\alpha-1) n}+\varepsilon^{1+m(\alpha-1)(n-p) /(p-1)}\right), \quad \text { and } \\
\left\|\nabla W_{\varepsilon}\right\|_{L^{m}(\Omega)}^{m} \leq K \varepsilon^{\alpha(n-m)-n+1}, \quad \text { for } m \in[1, p]
\end{gathered}
$$

where $K$ denotes a constant independent of $\varepsilon$. Consequently, we conclude that

$$
W_{\varepsilon} \rightarrow 0 \text { in } W^{1, p}(\Omega)-\text { weak as } \varepsilon \rightarrow 0 \text {, if } \alpha=(n-1) /(n-p) .
$$

Let us consider the functions

$$
\psi_{\varepsilon}=v-W_{\varepsilon}\left(H\left(x, v^{+}\right)+v^{-}\right)
$$

where $v \in C_{0}^{\infty}(\Omega), W_{\varepsilon}$ is the function defined by (15) and $H(x, \tau)$ is the solution of the functional equation (13). Note that, from (53), $\psi_{\varepsilon} \geq 0$ on $S_{\varepsilon}$, and thus it belongs to $K_{\varepsilon}$.

We now take $\psi=\psi_{\varepsilon}$ defined by (19) as a test function in (7) and pass to the limit when $\varepsilon \rightarrow 0$. On account of (10), (18) and the fact that $\left|G_{\varepsilon}\right| \rightarrow 0$ as $\varepsilon \rightarrow 0$, we deduce

$$
\lim _{\varepsilon \rightarrow 0} \int_{\Omega_{\varepsilon}} f\left(\psi_{\varepsilon}-u_{\varepsilon}\right) d x=\int_{\Omega} f(v-u) d x .
$$

Let us show that

$$
\begin{aligned}
& \lim _{\varepsilon \rightarrow 0}\left(\int_{\Omega_{\varepsilon}}\left|\nabla \psi_{\varepsilon}\right|^{p-2} \nabla \psi_{\varepsilon} \nabla\left(\psi_{\varepsilon}-u_{\varepsilon}\right) d x+\varepsilon^{-\kappa} \int_{S_{\varepsilon}} \sigma\left(x, \psi_{\varepsilon}\right)\left(\psi_{\varepsilon}-u_{\varepsilon}\right) d s\right) \\
\leq & \int_{\Omega}|\nabla v|^{p-2} \nabla v \nabla(v-u) d x+\int_{\gamma} g(x, v)(v-u) d \hat{x},
\end{aligned}
$$

where the function $g$ is given by (12a). In order to do that, we take into account (9), (10) and (18), and apply Lemma 2 with $\eta_{\varepsilon} \equiv-W_{\varepsilon} \widehat{H}$ and $\varphi=\varphi_{\varepsilon} \equiv v-W_{\varepsilon} \widehat{H}-\mathcal{P}_{\varepsilon} u_{\varepsilon}, \mathcal{P}_{\varepsilon} u_{\varepsilon}$ being the extension defined by $(10)$ and $\widehat{H} \equiv H\left(x, v^{+}\right)+v^{-}$. Thus, we obtain

$$
\lim _{\varepsilon \rightarrow 0} \int_{\Omega_{\varepsilon}}\left|\nabla \psi_{\varepsilon}\right|^{p-2} \nabla \psi_{\varepsilon} \nabla\left(\psi_{\varepsilon}-u_{\varepsilon}\right) d x=\lim _{\varepsilon \rightarrow 0}\left(L_{\varepsilon}^{1}-L_{\varepsilon}^{2}\right)
$$


where

$$
L_{\varepsilon}^{1} \equiv \int_{\Omega_{\varepsilon}}|\nabla v|^{p-2} \nabla v \nabla\left(v-W_{\varepsilon} \widehat{H}-u_{\varepsilon}\right) d x
$$

and

$$
L_{\varepsilon}^{2} \equiv \int_{\Omega_{\varepsilon}}\left|\nabla\left(W_{\varepsilon} \widehat{H}\right)\right|^{p-2} \nabla\left(W_{\varepsilon} \widehat{H}\right) \nabla\left(v-W_{\varepsilon} \widehat{H}-u_{\varepsilon}\right) d x .
$$

On account of (10), (18) and the fact that $\left|G_{\varepsilon}\right| \rightarrow 0$ as $\varepsilon \rightarrow 0$, we have

$$
\lim _{\varepsilon \rightarrow 0} L_{\varepsilon}^{1}=\int_{\Omega}|\nabla v|^{p-2} \nabla v \nabla(v-u) d x .
$$

On the other hand, from (16), (17), (9), and cumbersome computations, it follows that

$$
\lim _{\varepsilon \rightarrow 0} L_{\varepsilon}^{2}=\lim _{\varepsilon \rightarrow 0} \int_{\Omega_{\varepsilon}}|\widehat{H}|^{p-2}\left|\nabla W_{\varepsilon}\right|^{p-2} \nabla\left(W_{\varepsilon} \widehat{H}\right) \nabla \varphi_{\varepsilon} d x=\lim _{\varepsilon \rightarrow 0} \int_{\Omega_{\varepsilon}}\left|\nabla W_{\varepsilon}\right|^{p-2} \nabla W_{\varepsilon} \nabla\left(|\widehat{H}|^{p-2} \widehat{H} \varphi_{\varepsilon}\right) d x .
$$

Indeed, in the integral arising in $L_{\varepsilon}^{2}$ in $(24)$, we decompose the term $\left|\nabla\left(W_{\varepsilon} \widehat{H}\right)\right|^{p-2}$ in terms of powers of $\left|\nabla W_{\varepsilon} \widehat{H}\right|$ and $\left|W_{\varepsilon} \nabla \widehat{H}\right|$. Then, on account of (16), (17), (9), and the Hölder inequality for the suitable indices, we show that all the integrals in which the term $\left|W_{\varepsilon}\right|^{r}$ appears, for some $r \in(0, p]$, converge towards zero as $\varepsilon \rightarrow 0$. Thus, the first inequality in (26) holds. To prove the second equality in (26), we consider

$$
\int_{\Omega_{\varepsilon}}\left|\nabla W_{\varepsilon}\right|^{p-2} \nabla W_{\varepsilon} \nabla\left(|\widehat{H}|^{p-2} \widehat{H} \varphi_{\varepsilon}\right) d x=\int_{\Omega_{\varepsilon}}|\widehat{H}|^{p-2}\left|\nabla W_{\varepsilon}\right|^{p-2} \nabla\left(W_{\varepsilon} \widehat{H}\right) \nabla \varphi_{\varepsilon} d x+I_{\varepsilon}
$$

where $I_{\varepsilon}$ denotes a sum of integrals that converge towards zero because either it appears the term $\left|W_{\varepsilon}\right|^{r}$ as above or because the imbedding $W^{1, p}(\Omega) \subset L^{n p /(n-p)}(\Omega)$.

Moreover, by the properties of $H(x, z),|\widehat{H}|^{p-2} \widehat{H}=\left|H\left(x, v^{+}\right)\right|^{p-2} H\left(x, v^{+}\right)+\left|v^{-}\right|^{p-2} v^{-}$. Thus, using the definition of $W_{\varepsilon}$ and the Green formula, we get

$$
\lim _{\varepsilon \rightarrow 0} L_{\varepsilon}^{2}=\lim _{\varepsilon \rightarrow 0} \sum_{j \in \Upsilon_{\varepsilon}} \int_{\partial T_{\varepsilon / 4}^{j} \cup \partial G_{\varepsilon}^{j}}\left|\nabla w_{\varepsilon}^{j}\right|^{p-2} \partial_{\nu} w_{\varepsilon}^{j}\left(\left|H\left(x, v^{+}\right)\right|^{p-2} H\left(x, v^{+}\right)+\left|v^{-}\right|^{p-2} v^{-}\right) \varphi_{\varepsilon} d s .
$$

In order to compute (27), we use the explicit form of the normal derivatives of the auxiliary functions $w_{\varepsilon}^{j}$ given by

$$
\begin{aligned}
& \left.\left|\nabla w_{\varepsilon}^{j}\right|^{p-2} \partial_{\nu} w_{\varepsilon}^{j}\right|_{\partial G_{\varepsilon}^{j}}=\varepsilon^{-\frac{(n-1)(p-1)}{n-p}} \frac{\mathcal{D}_{n, p}}{\left(1-\alpha_{\varepsilon}\right)^{p-1}}, \text { and } \\
& \left.\left|\nabla w_{\varepsilon}^{j}\right|^{p-2} \partial_{\nu} w_{\varepsilon}^{j}\right|_{\partial T_{\varepsilon / 4}^{j}}=-\frac{2^{2 n-2} \mathcal{A}_{n, p}}{\omega_{n}\left(1-\alpha_{\varepsilon}\right)^{p-1}},
\end{aligned}
$$


where $\alpha_{\varepsilon} \rightarrow 0$ as $\varepsilon \rightarrow 0$. Thus, on account of the definitions of $\varphi_{\varepsilon}$ and $W_{\varepsilon},(28)$, (18) and (10), we apply Lemma 3 and have

$$
\begin{aligned}
& \lim _{\varepsilon \rightarrow 0} \sum_{j \in \Upsilon_{\varepsilon}} \int_{\partial T_{\varepsilon / 4}^{j}}\left|\nabla w_{\varepsilon}^{j}\right|^{p-2} \partial_{\nu} w_{\varepsilon}^{j}\left(\left|H\left(x, v^{+}\right)\right|^{p-2} H\left(x, v^{+}\right)+\left.\left|v^{-}\right|\right|^{p-2} v^{-}\right) \varphi_{\varepsilon} d s \\
= & -\mathcal{A}_{n, p} \int_{\gamma}\left(\left|H\left(x, v^{+}\right)\right|^{p-2} H\left(x, v^{+}\right)+\left|v^{-}\right|{ }^{p-2} v^{-}\right)(v-u) d \hat{x} .
\end{aligned}
$$

On the other hand, using again the definitions of $\varphi_{\varepsilon}$ and $W_{\varepsilon}$ and the fact that $v^{-}\left(v^{+}-\right.$ $\left.H\left(x, v^{+}\right)\right)=0, u_{\varepsilon} \geq 0$ on $\partial G_{\varepsilon}^{j}$ and (28), we obtain

$$
\begin{aligned}
& \lim _{\varepsilon \rightarrow 0} \sum_{j \in \Upsilon_{\varepsilon}} \int_{\partial G_{\varepsilon}^{j}}\left|\nabla w_{\varepsilon}^{j}\right|^{p-2} \partial_{\nu} w_{\varepsilon}^{j}\left(\left|H\left(x, v^{+}\right)\right|^{p-2} H\left(x, v^{+}\right)+\left|v^{-}\right| p^{-2} v^{-}\right) \varphi_{\varepsilon} d s \\
\geq & \lim _{\varepsilon \rightarrow 0} \frac{\varepsilon^{-\kappa} \mathcal{D}_{n, p}}{\left(1-\alpha_{\varepsilon}\right)^{p-1}} \int_{S_{\varepsilon}}\left|H\left(x, v^{+}\right)\right|^{p-2} H\left(x, v^{+}\right)\left(v^{+}-H\left(x, v^{+}\right)-u_{\varepsilon}\right) d s .
\end{aligned}
$$

Now, taking into account that $H$ is the solution of the equation (13) and using (19), (15), the Hölder inequality, (9), and the size of $S_{\varepsilon}$ we get

$$
\begin{aligned}
& \left.\left|\varepsilon^{-\kappa} \int_{S_{\varepsilon}} \sigma\left(x, \psi_{\varepsilon}\right)\left(\psi_{\varepsilon}-u_{\varepsilon}\right) d s-\frac{\varepsilon^{-\kappa} \mathcal{D}_{n, p}}{\left(1-\alpha_{\varepsilon}\right)^{p-1}} \int_{S_{\varepsilon}}\right| H\left(x, v^{+}\right)\right|^{p-2} H\left(x, v^{+}\right)\left(v^{+}-H\left(x, v^{+}\right)-u_{\varepsilon}\right) d s \mid \\
& \leq K \alpha_{\varepsilon} \varepsilon^{-\kappa} \int_{S_{\varepsilon}}\left|v^{+}-H\left(x, v^{+}\right)-u_{\varepsilon}\right| d s \leq K \alpha_{\varepsilon} \varepsilon^{-\kappa}\left[\left|S_{\varepsilon}\right|+\left|S_{\varepsilon}\right|^{(p-1) / p}\left\|u_{\varepsilon}\right\|_{L^{p}\left(S_{\varepsilon}\right)}\right] \rightarrow 0 \text { as } \varepsilon \rightarrow 0 .
\end{aligned}
$$

Therefore, gathering (22), (25), (27), (29), (30) and (31), where $L_{\varepsilon}^{1}$ and $L_{\varepsilon}^{2}$ are defined by (23) and (24) respectively, yields (21).

Finally, we use (20) and (21) to pass to the limit in (7), as $\varepsilon \rightarrow 0$, with $\psi=\psi_{\varepsilon}$ defined by (19), and obtain that the limit function $u$ satisfies the following inequality

$$
\int_{\Omega}|\nabla v|^{p-2} \nabla v \nabla(v-u) d x+\int_{\gamma} g(x, v)(v-u) d \hat{x} \geq \int_{\Omega} f(v-u) d x, \quad \forall v \in W^{1, p}(\Omega, \partial \Omega) .
$$

As usual, taking $v=u \pm \lambda \phi$ in (32) where $\phi \in W^{1, p}(\Omega, \partial \Omega)$ and passing to the limit as $\lambda \rightarrow+0$, we obtain that $u$ satisfies the integral identity (14), which concludes the proof for $\kappa=(n-1)(p-1) /(n-p)$.

For the cases $\kappa<(n-1)(p-1) /(n-p)$ and $\kappa>(n-1)(p-1) /(n-p)$, we rewrite the proof of $\kappa=(n-1)(p-1) /(n-p)$ with suitable modifications; we briefly outline the main differences here.

Second step: $\kappa<(n-1)(p-1) /(n-p)$ 
We now take in (7) the test function $\psi=\psi_{\varepsilon}=v-W_{\varepsilon} v^{-} \in K_{\varepsilon}$, where $v \in C_{0}^{\infty}(\Omega)$ and $W_{\varepsilon}$ is the function defined by (15), and pass to the limit when $\varepsilon \rightarrow 0$. Using (15), (3) and (9) and computing $\left|S_{\varepsilon}\right|$, it follows that

$$
\begin{aligned}
& \quad\left|\varepsilon^{-\kappa} \int_{S_{\varepsilon}} \sigma\left(x, \psi_{\varepsilon}\right)\left(\psi_{\varepsilon}-u_{\varepsilon}\right) d s\right|=\left|\varepsilon^{-\kappa} \int_{S_{\varepsilon}} \sigma\left(x, v^{+}\right)\left(v^{+}-u_{\varepsilon}\right) d s\right| \\
& \leq K \varepsilon^{-\kappa}\left[\left|S_{\varepsilon}\right|+\left|S_{\varepsilon}\right|^{(p-1) / p}\left\|u_{\varepsilon}\right\|_{L^{p}\left(S_{\varepsilon}\right)}\right] \leq K\left[\varepsilon^{(\alpha-1)(n-1)-\kappa}+\varepsilon^{((\alpha-1)(n-1)-\kappa)(p-1) / p}\right],
\end{aligned}
$$

which converges towards zero as $\varepsilon \rightarrow 0$. Moreover, (20) also holds. Let us show that $\lim _{\varepsilon \rightarrow 0} \int_{\Omega_{\varepsilon}}\left|\nabla \psi_{\varepsilon}\right|^{p-2} \nabla \psi_{\varepsilon} \nabla\left(\psi_{\varepsilon}-u_{\varepsilon}\right) d x \leq \int_{\Omega}|\nabla v|^{p-2} \nabla v \nabla(v-u) d x+\mathcal{A}_{n, p} \int_{\gamma}\left|v^{-}\right|^{p-2} v^{-}(v-u) d \hat{x}$.

On account of (17) and (10), we apply Lemma 2 with $\eta_{\varepsilon} \equiv-W_{\varepsilon} v^{-}$and $\varphi=\varphi_{\varepsilon} \equiv$ $v-W_{\varepsilon} v^{-}-\mathcal{P}_{\varepsilon} u_{\varepsilon}$. Thus, we obtain (22) where $L_{\varepsilon}^{1}$ and $L_{\varepsilon}^{2}$ are now replaced by

$$
L_{\varepsilon}^{1} \equiv \int_{\Omega_{\varepsilon}}|\nabla v|^{p-2} \nabla v \nabla\left(v-W_{\varepsilon} v^{-}-u_{\varepsilon}\right) d x
$$

and

$$
L_{\varepsilon}^{2} \equiv \int_{\Omega_{\varepsilon}}\left|\nabla\left(W_{\varepsilon} v^{-}\right)\right|^{p-2} \nabla\left(W_{\varepsilon} v^{-}\right) \nabla\left(v-W_{\varepsilon} v^{-}-u_{\varepsilon}\right) d x .
$$

By (18), (10) and the fact that $\left|G_{\varepsilon}\right| \rightarrow 0,(25)$ also holds. Moreover, using (17), (9), the definition of $W_{\varepsilon}$, the fact that $v^{-} v^{+}=0$ and the Green formula, we get

$$
\lim _{\varepsilon \rightarrow 0} L_{\varepsilon}^{2}=\lim _{\varepsilon \rightarrow 0} \sum_{j \in \Upsilon_{\varepsilon}} \int_{\partial T_{\varepsilon / 4}^{j} \cup \partial G_{\varepsilon}^{j}}\left|\nabla w_{\varepsilon}^{j}\right|^{p-2} \partial_{\nu} w_{\varepsilon}^{j}\left|v^{-}\right|^{p-2} v^{-}\left(v-W_{\varepsilon} v^{-}-u_{\varepsilon}\right) d s .
$$

Then, from (28), Lemma 3, (18) and (10), we have

$$
\lim _{\varepsilon \rightarrow 0} \sum_{j \in \Upsilon_{\varepsilon}} \int_{\partial T_{\varepsilon / 4}^{j}}\left|\nabla w_{\varepsilon}^{j}\right|^{p-2} \partial_{\nu} w_{\varepsilon}^{j}\left|v^{-}\right|^{p-2} v^{-}\left(v-W_{\varepsilon} v^{-}-u_{\varepsilon}\right) d s=-\mathcal{A}_{n, p} \int_{\gamma}\left|v^{-}\right|^{p-2} v^{-}(v-u) d \hat{x} .
$$

Besides, by the definition of $W_{\varepsilon}$ (cf. (15)) and the fact that $v^{-} v^{+}=0, u_{\varepsilon} \geq 0$ on $\partial G_{\varepsilon}^{j}$ and (28), we obtain

$$
\sum_{j \in \Upsilon_{\varepsilon}} \int_{\partial G_{\varepsilon}^{j}}\left|\nabla w_{\varepsilon}^{j}\right|^{p-2} \partial_{\nu} w_{\varepsilon}^{j}\left|v^{-}\right|^{p-2} v^{-}\left(v-W_{\varepsilon} v^{-}-u_{\varepsilon}\right) d s \geq 0 .
$$

Gathering (22), (25), (37), (38) and (39), where $L_{\varepsilon}^{1}$ and $L_{\varepsilon}^{2}$ are now defined by (35) and (36) respectively, yields (34).

Thus, using (20), (33) and (34), we pass to the limit in (7), as $\varepsilon \rightarrow 0$ with $\psi=\psi_{\varepsilon}=$ $v-W_{\varepsilon} v^{-}$, and obtain that the limit function $u$ satisfies inequality (32) where $g(x, u)$ 
is now given by $(12 \mathrm{~b})$. From here, the argument at the end of the proof of the case $\kappa=(n-1)(p-1) /(n-p)$ leads us to state that $u$ satisfies the integral identity (14), which concludes the proof for $\kappa<(n-1)(p-1) /(n-p)$.

Third step: $\kappa>(n-1)(p-1) /(n-p)$

We now take in (7) the test function $\psi=\psi_{\varepsilon}=v-W_{\varepsilon} v \in K_{\varepsilon}$, where $v \in C_{0}^{\infty}(\Omega)$ and $W_{\varepsilon}$ is the function defined by (15), and pass to the limit when $\varepsilon \rightarrow 0$. On account of the properties of $W_{\varepsilon}$ and $\sigma$,

$$
\sigma\left(x, \psi_{\varepsilon}\right)=0 \text { on } S_{\varepsilon} .
$$

Moreover, (20) also holds. Let us show that

$\lim _{\varepsilon \rightarrow 0} \int_{\Omega_{\varepsilon}}\left|\nabla \psi_{\varepsilon}\right|^{p-2} \nabla \psi_{\varepsilon} \nabla\left(\psi_{\varepsilon}-u_{\varepsilon}\right) d x=\int_{\Omega}|\nabla v|^{p-2} \nabla v \nabla(v-u) d x+\mathcal{A}_{n, p} \int_{\gamma}|v|^{p-2} v(v-u) d \hat{x}$.

On account of (17) and (10), we apply Lemma 2 with $\eta_{\varepsilon} \equiv-W_{\varepsilon} v$ and $\varphi=\varphi_{\varepsilon} \equiv$ $v-W_{\varepsilon} v-\mathcal{P}_{\varepsilon} u_{\varepsilon}$. Thus, we obtain (22) where $L_{\varepsilon}^{1}$ and $L_{\varepsilon}^{2}$ are now replaced by

$$
L_{\varepsilon}^{1} \equiv \int_{\Omega_{\varepsilon}}|\nabla v|^{p-2} \nabla v \nabla\left(v-W_{\varepsilon} v-u_{\varepsilon}\right) d x
$$

and

$$
L_{\varepsilon}^{2} \equiv \int_{\Omega_{\varepsilon}}\left|\nabla\left(W_{\varepsilon} v\right)\right|^{p-2} \nabla\left(W_{\varepsilon} v\right) \nabla\left(v-W_{\varepsilon} v-u_{\varepsilon}\right) d x .
$$

By (18), (10) and the fact that $\left|G_{\varepsilon}\right| \rightarrow 0$, (25) also holds. Moreover, using (17), (9), the definition of $W_{\varepsilon}$ and the Green formula, we get

$$
\lim _{\varepsilon \rightarrow 0} L_{\varepsilon}^{2}=\lim _{\varepsilon \rightarrow 0} \sum_{j \in \Upsilon_{\varepsilon}} \int_{\partial T_{\varepsilon / 4}^{j} \cup \partial G_{\varepsilon}^{j}}\left|\nabla w_{\varepsilon}^{j}\right|^{p-2} \partial_{\nu} w_{\varepsilon}^{j}|v|^{p-2} v\left(v-W_{\varepsilon} v-u_{\varepsilon}\right) d s .
$$

Now, from (28), Lemma 3 and (10), we have

$$
\lim _{\varepsilon \rightarrow 0} \sum_{j \in \Upsilon_{\varepsilon}} \int_{\partial T_{\varepsilon / 4}^{j}}\left|\nabla w_{\varepsilon}^{j}\right|^{p-2} \partial_{\nu} w_{\varepsilon}^{j}|v|^{p-2} v\left(v-W_{\varepsilon} v-u_{\varepsilon}\right) d s=-\mathcal{A}_{n, p} \int_{\gamma}|v|^{p-2} v(v-u) d \hat{x} .
$$

Besides, by (15), (28) and (9), and the fact that $\left|S_{\varepsilon}\right| \leq K \varepsilon^{(\alpha-1)(n-1)}$, it follows that

$$
\begin{aligned}
& \left.\quad\left|\sum_{j \in \Upsilon_{\varepsilon}} \int_{\partial G_{\varepsilon}^{j}}\right| \nabla w_{\varepsilon}^{j}\right|^{p-2} \partial_{\nu} w_{\varepsilon}^{j}|v|^{p-2} v\left(v-W_{\varepsilon} v-u_{\varepsilon}\right) d s|=| \frac{\varepsilon^{-\alpha(p-1)} \mathcal{D}_{n, p}}{\left(1-\alpha_{\varepsilon}\right)^{p-1}} \int_{S_{\varepsilon}}|v|^{p-2} v u_{\varepsilon} d s \mid \\
& \leq K \varepsilon^{-\alpha(p-1)}\left|S_{\varepsilon}\right|^{(p-1) / p}\left\|u_{\varepsilon}\right\|_{L^{p}\left(S_{\varepsilon}\right)} \leq K \varepsilon^{[\kappa-\alpha(p-1)] / p} \rightarrow 0 \quad \text { as } \varepsilon \rightarrow 0 .
\end{aligned}
$$

Gathering (22), (25), (44), (45) and (46), where $L_{\varepsilon}^{1}$ and $L_{\varepsilon}^{2}$ are now defined by (42) and (43) respectively, yields (41). 
Thus, using (20), (40) and (41), we pass to the limit in (7), as $\varepsilon \rightarrow 0$ with $\psi=$ $\psi_{\varepsilon}=v-W_{\varepsilon} v$, and obtain that the limit function $u$ satisfies inequality (32) where $g(x, u)$ is now given by (12c). From here, the argument at the end of the proof of the case $\kappa=(n-1)(p-1) /(n-p)$ leads us to state that $u$ satisfies the integral identity (14), which concludes the proof for $\kappa>(n-1)(p-1) /(n-p)$.

\section{Other sizes for perforations}

In this section, we deal with sizes of cavities larger or smaller than the critical size. For the case of big cavities and critical relations for the adsorption parameter, that is $\alpha \in(1,(n-1) /(n-p))$ and $\kappa=(\alpha-1)(n-1)$, the homogenized problem is a unilateral problem with a strange term dependent on $\sigma$ (cf. (47) and (48a)). For the case of big cavities and small adsorption, that is $\alpha \in(1,(n-1) /(n-p))$ and $\kappa<(\alpha-1)(n-1)$, the homogenized problem is a unilateral problem which ignores the adsorption parameter (cf. (47) and (48b)). For the case of large sizes of cavities and adsorption, the solution of (4) vanishes asymptotically on the manifold $\gamma$ and the homogenized problem is the Dirichlet problem in $\Omega^{+} \cup \Omega^{-}$for the $p$-Laplace operator (cf. (50)). Finally, for the case of very small cavities, the solution ignores the perforations and the adsorption parameter; now the homogenized problem is the Dirichlet problem in $\Omega$ (cf. (51)).

Theorem 2. Let $\alpha \in(1,(n-1) /(n-p))$ with $n \geq 3, p \in[2, n)$, and let $u_{\varepsilon}$ be the weak solution of (4). Then, the limit of $\mathcal{P}_{\varepsilon} u_{\varepsilon}$ in (10), $u$, is the weak solution of problem

$$
\left\{\begin{array}{l}
-\Delta_{p} u=f \quad \text { in } \Omega^{+} \cup \Omega^{-}, \quad u=0 \quad \text { on } \partial \Omega \\
{[u]=0, \quad u \geq 0, \quad h-\left[|\nabla u|^{p-2} \partial_{x_{1}} u\right] \geq 0, \quad u\left(h-\left[|\nabla u|^{p-2} \partial_{x_{1}} u\right]\right)=0 \quad \text { on } \gamma}
\end{array}\right.
$$

where the function $h$ is given by

$$
\begin{array}{ll}
h(x, u)=C_{0}^{n-1} \omega_{n} \sigma(x, u) & \text { if } \kappa=(\alpha-1)(n-1), \\
h(x, u)=0 & \text { if } \kappa<(\alpha-1)(n-1) .
\end{array}
$$

Sketch of the proof. The variational formulation of (47) reads: find $u \in K_{0}$ such that

$$
\int_{\Omega}|\nabla u|^{p-2} \nabla u \nabla \phi d x+\int_{\gamma} h(x, u) \phi d \hat{x} \geq \int_{\Omega} f \phi d x, \quad \forall \phi \in K_{0},
$$

where $K_{0}=\left\{\phi \in W^{1, p}(\Omega, \partial \Omega): \phi \geq 0\right.$ a.e. on $\left.\gamma\right\}$. Using Lemma 5, (9) and the fact that $u_{\varepsilon}^{-}=0$ on $S_{\varepsilon}$, we deduce that

$$
\frac{1}{\varepsilon}\left\|\mathcal{P}_{\varepsilon} u_{\varepsilon}^{-}\right\|_{L^{r}\left(\Pi_{\varepsilon}\right)}^{r} \rightarrow 0 \text { when } \varepsilon \rightarrow 0, \quad \text { for } r \in[2, p]
$$


where $\Pi_{\varepsilon}=\Omega \cap\left\{-\varepsilon / 2<x_{1}<\varepsilon / 2\right\}$. Now, since $\mathcal{P}_{\varepsilon} u_{\varepsilon}^{-}$converge towards $u^{-}$in $L^{2}(\gamma)$, by Lemma 4 and (9), we conclude that $u^{-}$vanishes on $\gamma$ and $u \in K_{0}$.

To prove that $u$ satisfies (49), we pass to the limit in (7) with $\psi=v \in \mathcal{H}_{\gamma}$, where $\mathcal{H}_{\gamma}=\left\{\phi \in W^{1, p}(\Omega, \partial \Omega): \phi \geq 0\right.$ in some neighbourhood of $\left.\gamma\right\}$. From (10) we obtain (20) and (41) with $\psi_{\varepsilon} \equiv v \in \mathcal{H}_{\gamma}$ and $\mathcal{A}_{n, p} \equiv 0$. Besides, following the technique in Theorem 4.1 in [7] and Theorems 5.1 and 6.1 in [5] with the suitable modifications, we prove

$$
\lim _{\varepsilon \rightarrow 0} \varepsilon^{-\kappa} \int_{S_{\varepsilon}} \sigma(x, v)\left(v-u_{\varepsilon}\right) d s=\int_{\gamma} h(x, v)(v-u) d \hat{x},
$$

where the function $h$ is defined by (48); cf. also (33) when $h=0$. Thus, since $\mathcal{H}_{\gamma}$ is dense in $K_{0}, u$ satisfies (49), which concludes the proof.

Theorem 3. Let $\alpha \in(1,(n-1) /(n-p)), \kappa>(\alpha-1)(n-1)$ with $n \geq 3, p \in[2, n)$, and let $u_{\varepsilon}$ be the weak solution of (4). Then, the limit of $\mathcal{P}_{\varepsilon} u_{\varepsilon}$ in (10), $u$, is the weak solution of the Dirichlet problem in $\Omega^{+} \cup \Omega^{-}$

$$
-\Delta_{p} u=f \quad \text { in } \Omega^{+} \cup \Omega^{-}, \quad u=0 \quad \text { on } \partial \Omega \cup \gamma
$$

Sketch of the proof. Let us take the test function $\psi=v$ in $(7)$, where $v \in C_{0}^{\infty}\left(\Omega^{ \pm}\right)$to obtain that $-\Delta_{p} u=f$ in $\mathcal{D}^{\prime}\left(\Omega^{ \pm}\right)$. Besides, using Lemma 5 and (9), we deduce that

$$
\frac{1}{\varepsilon}\left\|\mathcal{P}_{\varepsilon} u_{\varepsilon}\right\|_{L^{r}\left(\Pi_{\varepsilon}\right)}^{r} \rightarrow 0 \text { when } \varepsilon \rightarrow 0, \quad \text { for } r \in[2, p] .
$$

Then, since $\mathcal{P}_{\varepsilon} u_{\varepsilon}$ converge towards $u$ in $L^{2}(\gamma)$, by Lemma 4 and (9), we conclude that $u$ vanishes on $\gamma$ and the theorem holds.

Theorem 4. Let $\alpha>(n-1) /(n-p), \kappa \in \mathbb{R}$ with $n \geq 3, p \in[2, n)$, and let $u_{\varepsilon}$ be the weak solution of (4). Then, the limit of $\mathcal{P}_{\varepsilon} u_{\varepsilon}$ in (10), $u$, is the weak solution of the Dirichlet problem

$$
-\Delta_{p} u=f \quad \text { in } \Omega, \quad u=0 \quad \text { on } \partial \Omega .
$$

Sketch of the proof. We take in (7) the test function $\psi=\psi_{\varepsilon}=v-W_{\varepsilon} v \in K_{\varepsilon}$, where $v \in C_{0}^{\infty}(\Omega)$ and $W_{\varepsilon}$ is the function defined by (15), and pass to the limit when $\varepsilon \rightarrow 0$. Considering (40) and the fact that now $W_{\varepsilon}$ converges towards zero in $W^{1, p}(\Omega)$ (cf. (17)), we have (20) and (41) with $\mathcal{A}_{n, p} \equiv 0$. Now, we proceed as in the proof of Theorem 1 to show that $\mathrm{u}$ satisfies the weak formulation of the Dirichlet problem (51).

Remark 1. The nonlinear function $\sigma$ considered, cf. (1)-(3), allows us to provide a general framework for results and proofs. Actually, the strong monotonicity outlined in (2) can be changed by the weaker hypothesis of strict monotonicity or only monotonicity depending on the relations for parameters. This can be seen in a simple way when verifying proofs. 
Also, it should be noticed that the nonlinear adsorption isotherms often used in the literature (cf., e.g., [10] and [19]) are of the form $\sigma(x, u)=g(u)$ with $g$ a positive strictly increasing function in $[0, \infty)$. In this connection, we also note that certain proofs can be adapted for functions $\sigma$ both with less smoothness or increasing requirements. We refer to [1] for explicit definitions of $\sigma$ arising in models from ecology, hydrogeology or chemical reactions, for comments on possible extensions when $u \leq 0$, and for further references.

\section{Appendix}

In this section, we introduce some results useful for proofs. The first result provides the existence and uniqueness of the solution of the functional equation (13) arising in the homogenized problem (11)-(12a) while the second one simplifies the computations throughout the paper. The proofs of both results can be found in [5] (cf. Propositions 2.2 and 2.3 , respectively).

Lemma 1. Let $p \geq 2$. Let $\varrho$ be a strictly positive constant and let $\sigma$ be the function $\sigma(x, u)$ defined from $\bar{\Omega} \times \mathbb{R}$ into $\mathbb{R}$ which is assumed to be a continuously differentiable function in $\bar{\Omega} \times \mathbb{R}$ satisfying (1)-(2). Then, the equation

$$
|H|^{p-2} H=\varrho \sigma(x, \tau-H)
$$

has a unique solution $H(x, \tau)$ which is a continuously differentiable function in $\bar{\Omega} \times(\mathbb{R} \backslash\{0\})$ and continuous in $\bar{\Omega} \times \mathbb{R}$, and satisfies $H(x, 0)=0$ and

$$
\begin{gathered}
\left(|H(x, u)|^{p-2} H(x, u)-|H(x, v)|^{p-2} H(x, v)\right)(u-v) \geq \widetilde{k}_{1}|u-v|^{p}, \\
|H(x, u)| \leq|u|
\end{gathered}
$$

for all $x \in \bar{\Omega}, u, v \in \mathbb{R}$ and a certain constant $\widetilde{k}_{1}>0$.

Lemma 2. Let $p \geq 2$. Let $v \in W^{1, \infty}(\Omega), \varphi \in W^{1, p}(\Omega, \partial \Omega)$ and $\eta_{\varepsilon} \in W^{1, p}(\Omega, \partial \Omega)$ such that $\left\|\nabla \eta_{\varepsilon}\right\|_{L^{m}(\Omega)} \rightarrow 0$, as $\varepsilon \rightarrow 0$, for $m \in[1, p)$. Then,

$$
\lim _{\varepsilon \rightarrow 0} \int_{\Omega_{\varepsilon}}\left(\left|\nabla\left(v+\eta_{\varepsilon}\right)\right|^{p-2} \nabla\left(v+\eta_{\varepsilon}\right)-|\nabla v|^{p-2} \nabla v\right) \nabla \varphi d x=\lim _{\varepsilon \rightarrow 0} \int_{\Omega_{\varepsilon}}\left|\nabla \eta_{\varepsilon}\right|^{p-2} \nabla \eta_{\varepsilon} \nabla \varphi d x .
$$

In addition, (54) also holds in the case where $\varphi$ depends on $\varepsilon$, namely $\varphi \equiv \varphi_{\varepsilon}$, with $\left\|\nabla \varphi_{\varepsilon}\right\|_{L^{p}(\Omega)}$ bounded independently of $\varepsilon$.

Finally, we introduce the following auxiliary estimates where the constant $K$ does not depend on $\varepsilon$ nor on the functions $w$ : 
Lemma 3. Let $P_{\varepsilon}^{j}$ be the center of the ball $G_{\varepsilon}^{j}$ and let $T_{\varepsilon / 4}^{j}$ denote the ball of radius $\varepsilon / 4$ with center $P_{\varepsilon}^{j}, j \in \Upsilon_{\varepsilon}$. Then,

$$
\left|\sum_{j \in \Upsilon_{\varepsilon}} \int_{\partial T_{\varepsilon / 4}^{j}} w d s-2^{2-2 n} \omega_{n} \int_{\gamma} w d \hat{x}\right| \leq K \varepsilon^{1 / 2}\|w\|_{H^{1}(\Omega)}, \quad w \in H_{0}^{1}(\Omega) .
$$

See Lemma 1 in [16] for the proof.

Lemma 4. Let $\Pi_{\varepsilon}=\Omega \cap\left\{-\varepsilon / 2<x_{1}<\varepsilon / 2\right\}$. Then,

$$
\left|\frac{1}{\varepsilon} \int_{\Pi_{\varepsilon}} w^{2} d x-\int_{\gamma} w^{2} d \hat{x}\right| \leq K \varepsilon^{1 / 2}\|\nabla w\|_{L^{2}(\Omega)}^{2}, \quad w \in H_{0}^{1}(\Omega) .
$$

See Lemma 2.6 in [8] for precise references for the proof.

Lemma 5. Let $\Pi_{\varepsilon}=\Omega \cap\left\{-\varepsilon / 2<x_{1}<\varepsilon / 2\right\}$. Let $w \in W^{1, p}(\Omega), 2 \leq p<n$. Then,

$$
\begin{gathered}
\|w\|_{L^{p}\left(G_{\varepsilon}\right)}^{p} \leq K\left(a_{\varepsilon}\|w\|_{L^{p}\left(S_{\varepsilon}\right)}^{p}+a_{\varepsilon}^{p}\|\nabla w\|_{L^{p}\left(G_{\varepsilon}\right)}^{p}\right), \quad \text { and } \\
\|w\|_{L^{p}\left(\Pi_{\varepsilon} \backslash G_{\varepsilon}\right)}^{p} \leq K\left(a_{\varepsilon}^{1-n} \varepsilon^{n}\|w\|_{L^{p}\left(S_{\varepsilon}\right)}^{p}+a_{\varepsilon}^{p-n} \varepsilon^{n}\|\nabla w\|_{L^{p}(\Omega)}^{p}\right) .
\end{gathered}
$$

See Theorem 5.1 in [8] and Lemma 2.6 in [5] related to the proofs of the first and the second inequality respectively.

Acknowledgments: This work has been partially supported by the Spanish grant MINECO:MTM2013-44883-P.

\section{References}

[1] Brillard A, Gómez D, Lobo M, Pérez E, Shaposhnikova TA (2016) Boundary homogenization in perforated domains for adsorption problems with an advection term. Appl Anal 95:1517-1533.

[2] Cioranescu D, Murat F (1997) A strange term coming from nowhere. In: Topics in the Mathematical Modelling of Composite Materials. Progr. Nonlinear Differential Equations Appl. 31, Birkäuser, pp. 45-93.

[3] Díaz JI, Gómez-Castro D, Podolskii AV, Shaposhnikova TA (2017) Homogenization of variational inequalities of Signorini type for the $p$-Laplacian in perforated domains when $p \in(1,2)$. Dokl Math 95:151-156.

[4] Gómez D, Lobo M, Pérez E, Podolskii AV, Shaposhnikova TA (2014) Homogenization for the $p$-Laplace operator and nonlinear Robin boundary conditions in perforated media along manifolds. Dokl Math 89:11-15. 
[5] Gómez D, Lobo M, Pérez E, Podolskii AV, Shaposhnikova TA (2017) Unilateral problems for the $p$-Laplace operator in perforated media involving large parameters. ESAIM Control Optim Calc Var DOI: 10.1051/cocv/2017026.

[6] Gómez D, Lobo M, Pérez E, Shaposhnikova TA (2011) Averaging in variational inequalities with nonlinear restrictions along manifolds. CR Mecanique 339:406-410.

[7] Gómez D, Lobo M, Pérez E, Shaposhnikova TA (2013) Averaging of variational inequalities for the Laplacian with nonlinear restrictions along manifolds. Appl Anal $92: 218-237$.

[8] Gómez D, Pérez E, Shaposhnikova TA (2012) On homogenization of nonlinear Robin type boundary conditions for cavities along manifolds and associated spectral problems. Asymptot Anal 80:289-322.

[9] Gómez D, Pérez E, Shaposhnikova TA (2013) Spectral boundary homogenization problems in perforated domins with Robin boundary conditions and large parameters. In: Integral Methods in Science and Engineering. Progress in Numerical and Analytic Techniques, Birkhäuser, pp. 155-174.

[10] Hornung U (1997) Homogenization and Porous Media, Springer-Velag.

[11] Hornung U, Jäger W (1991) Diffusion, convection, adsorption, and reaction of chemicals in porous media. J Differential Equations 92:199-225.

[12] Hornung U, Showalter RE (1995) Elliptic-parabolic equations with hysteresis boundary conditions. SIAM J Math Anal 26:775-790.

[13] Jäger W, Neuss-Radu M, Shaposhnikova TA (2011) Scale limit of a variational inequality modeling diffusive flux in a domain with small holes and strong adsorption in case of a critical scaling. Dokl Math 83:204-208.

[14] Kaizu S (1989) The Poisson equation with semilinear boundary conditions in domains with many tiny holes. J. Fac. Sci. Univ. Tokyo Sect. IA Math. 36:43-86.

[15] Labani N, Picard C (1989) Homogenization of a nonlinear Dirichlet problem in a periodically perforated domain. In: Recent Advances in Nonlinear Elliptic and Parabolic Problems, Pitman Res. Notes Math. Ser. 208, Longman Sci. Tech., Harlow, pp. 294305.

[16] Lobo M, Oleinik OA, Perez ME, Shaposhnikova TA (1997) On homogenization of solutions of boundary value problem in domains, perforated along manifolds. Ann Scuola Norm Sup Pisa Cl Sci Ser 4 25:611-629. 
[17] Lobo M, Perez ME, Sukharev VV, Shaposhnikova TA (2011) Averaging of boundaryvalue problem in domain perforated along $(n-1)$-dimensional manifold with nonlinear third type boundary conditions on the boundary of cavities. Dokl Math 83:34-38.

[18] Marpeau F, Saad M (2006) Mathematical analysis of radionuclides displacement in porous media with nonlinear adsorption. J Differential Equations 228:412-439.

[19] Marsily G (1986) Quantitative Hydrogeolog: Groundwater Hydrology for Engineers, Academic Press.

[20] Shaposhnikova TA, Podolskii AV (2012) Homogenization limit for the boundary value problem with the $p$-Laplace operator and a nonlinear third boundary condition on the boundary of the holes in a perforated domain. Funct. Differ. Equ. 19:351-370.

[21] Showalter RE, Walkington NJ (1991) Diffusion of fluid in a fissured medium with microstructure. SIAM J Math Anal 22:1702-1722.

[22] Podolskii AV (2015) Solution continuation and homogenization of a boundary value problem for the $p$-Laplacian in a perforated domain with a nonlinear third boundary condition on the boundary of holes. Dokl Math 91:30-34.

[23] Zubova MN, Shaposhnikova TA (2007) On homogenization of variational inequalities with obstacles on $\varepsilon$-periodically situated along $n-1$ dimension manifold inclusions. Funct Differ Equ 14:123-143.

[24] Zubova MN, Shaposhnikova TA (2011) Homogenization of boundary value problems in perforated domains with the third boundary condition and the resulting change in the character of the nonlinearity in the problem. Differ Equ 47(1):78-90. 